\section{Tamoxifen treats symptoms of mania in patients with bipolar disorder}

The protein kinase $C(P K C)$ signaling cascade has been identified as a direct biochemical target for the treatment of mania. Preclinical studies and early clinical trials have shown that the anticancer agent tamoxifen, a relatively selective PKC inhibitor that can cross the blood-brain barrier, exerts an antimanic effect in a mouse model of mania. Zarate et al. have now examined the effect of tamoxifen in a double-blind pilot trial of 16 inpatients in the manic phase of bipolar disorder.

Study participants received flexible dosing of tamoxifen (20-140 mg; $n=8$ ) or placebo $(n=8)$ for 21 days. At the end of the study period, a significant difference was seen between the effects of tamoxifen and placebo on manic symptoms (Cohen's $d=1.08,95 \% \mathrm{Cl}$ $0.45-1.71 ; P=0.001)$. After 3 weeks of therapy, $63 \%$ of patients in the treatment group showed a clinical response-defined as at least a $50 \%$ reduction from baseline in Young Mania Rating Scale total score-compared with $13 \%$ of those receiving placebo $(P=0.12)$. Notably, patients receiving tamoxifen showed improvement in symptoms as early as 5 days after the start of treatment. Therapy was generally well tolerated.

The authors conclude that the results of this small pilot study are proof of the concept that direct inhibition of PKC could form the basis of fast-acting medications for the manic phase of bipolar disorder. Further studies in this setting are warranted.

Original article Zarate CA Jr et al. (2007) Efficacy of a protein kinase $\mathrm{C}$ inhibitor (tamoxifen) in the treatment of acute mania: a pilot study. Bipolar Disord 9: 561-570

\section{New drug for schizophrenia modulates glutamate transmission}

Antipsychotics commonly used in the treatment of schizophrenia target dopamine receptors, but there is growing evidence that altered glutamate transmission is important in the pathophysiology of the disease. Researchers have developed a new drug, dubbed LY2140023, which is converted to a selective agonist of metabotropic glutamate 2/3 (mGlu2/3) receptors once absorbed. In a mouse study, the active
mGlu2/3 receptor agonist was shown to inhibit phencyclidine-induced hyperlocomotion via a mechanism different to that of olanzapine. A double-blind, placebo-controlled phase II study was undertaken to test the clinical usefulness of LY2140023.

Patil et al. randomized 196 patients with schizophrenic psychopathology to receive either LY2140023 (40 mg twice daily), olanzapine (15 mg once daily) or placebo in a 3:1:2 ratio; 118 patients completed the planned 4 weeks of treatment. Positive and Negative Symptom Scale (PANSS) scores improved significantly from baseline in patients treated with LY2140023 and olanzapine $(P<0.001$ for both). The onset of efficacy was rapid in both groups, with significant effects apparent at 1 week $(P<0.05)$ and maintained until the end of the study period. LY2140023 was generally well-tolerated, and, notably, did not result in elevated prolactin levels, worsening of extrapyramidal symptoms or weight gain compared with placebo.

This study provides conclusive evidence of a role for glutamate in the pathophysiology of schizophrenia, and indicates that mGlu2/3 receptor agonism could be a valid therapeutic approach in the disease.

Original article Patil ST et al. (2007) Activation of mGlu2/3 receptors as a new approach to treat schizophrenia: a randomized phase 2 clinical trial. Nat Med 13: 1102-1107

\section{Targeting $T$ cells to prevent secondary damage following traumatic brain injury}

Nitrone radical scavengers have been shown to improve morphological and functional outcome in animal models of traumatic brain injury (TBI). Clausen et al. hypothesized that nonpenetrating nitrones act systemically by interacting with the peripheral immune system at the microvascular level to prevent the secondary injury cascade following TBI. To test this theory, they examined whether 2-sulfophenyl- $N$-tert-butyl-nitrone (S-PBN) modulated the trafficking of damaging components of the immune response in a rat model of TBI.

Immune cell infiltration was measured by flow cytometry and quantitative immunohistochemistry following ex vivo propagation of $T$ lymphocytes, neutrophils and cells of the macrophage-microglial lineage. A time-course study of leukocyte trafficking into the injured 